\title{
LUCA VERZELLONI
}

\section{Il lungo dibattito sui criteri di priorità negli uffici giudicanti e requirenti}

\begin{abstract}
SOMMARIO: 1.Introduzione. - 2.Le origini del dibattito. - 3.Il consolidarsi dell'indirizzo del CSM. 4.La "riscoperta" del dibattito. - 5. Il decreto del Tribunale ordinario di Brescia. - 6.Riflessioni conclusive.
\end{abstract}

\section{Introduzione.}

La giustizia italiana vive ormai da tempo una stagione di forte criticità. Negli ultimi trent'anni, diversi fattori hanno aggravato le difficoltà di funzionamento del sistema. In particolare, l'aumento dei tassi di litigiosità, la carenza di risorse umane, finanziarie e materiali, l'allungamento dei tempi di definizione dei procedimenti e il clima generale di sfiducia di cittadini e imprese hanno avuto un impatto diretto sull'amministrazione della giustizia italiana, tanto da rimettere in discussione la capacità degli uffici giudicanti e requirenti di garantire un servizio - come quello giudiziario - di fondamentale importanza per l'effettiva tutela dei diritti dei cittadini.

$\mathrm{Al}$ di là dei proclami e delle dichiarazioni programmatiche, l'emergere della cosiddetta "questione organizzativa" nella giustizia italiana è un fenomeno relativamente recente. I temi dell'organizzazione giudiziaria, infatti, si sono trasformati da ambito di nicchia - oggetto dell'interesse di un nucleo ristretto di studiosi ${ }^{1}$ e dell'iniziativa individuale di alcuni capi ufficio - a questione centrale per una moltitudine di operatori, ritenuta di fondamentale importanza per garantire la stessa sopravvivenza del sistema. In particolare, segnali di questo cambiamento sono l'aumento esponenziale di convegni, iniziative e pubblicazioni su questi argomenti, così come il diffondersi di concetti prima estranei al mondo della giustizia italiana, come: flussi di lavoro, efficienza, qualità del servizio, programmazione, soddisfazione dell'utenza, accountability, ecc.

Alla luce di questa premessa, il presente articolo intende ripercorrere e commentare il lungo dibattito sui criteri di priorità negli uffici giudicanti e requirenti, concentrandosi, in particolare, sul caso del Tribunale di Brescia. La tematica appare di estrema attualità, dal momento che risulta legata al sottodimensionamento delle piante organiche - sia del personale togato ${ }^{2}$ che, so-

\footnotetext{
' Per risalire alle origini degli studi sull'amministrazione giudiziaria italiana, si veda: Di FedErico, L'uso di strumenti elettronici nell'amministrazione della giustizia, 1966 e ID, Scienza dell'amministrazione e ordinamento giudiziario, 1975.

${ }^{2}$ In 9 distretti si registrano scoperture del personale togato superiori al 15\%: Ancona (17,03\%), Caltanisetta $(21,05 \%)$, Catanzaro $(22,86 \%)$, Messina $(17,58 \%)$, Palermo $(15,25 \%)$, Potenza $(21,60 \%)$, Reggio Calabria (18,01\%), Torino (16,13\%), Trento (compresa la sezione distaccata di Bolzano) $(16,19 \%)$.
} 
Archivio Penale 2014, n. 3

prattutto, amministrativo ${ }^{3}$ - che in questi anni si è ulteriormente aggravato, tanto da diventare un problema cronico del sistema italiano.

\section{Le origini del dibattito.}

Le origini del dibattito sui criteri di priorità possono essere fatte risalire fra la fine degli anni '80 e l'inizio degli anni '90. Il punto di partenza di questo dialogo a più voci è rappresentato dalla c.d. "circolare Pieri-Conti" (08 marzo 1989) ${ }^{4}$ e, in particolare, dalla successiva, più volte citata, c.d. "circolare Zagrebelsky" (16 novembre 1990), in cui l'allora Procuratore della Repubblica presso la Pretura di Torino stabiliva che: «'individuazione di criteri di priorità non contrasta con l'obbligo di cui all'art. 112 Cost., dal momento che il possibile mancato esercizio di un'azione penale tempestiva e adeguatamente preparata per tutte le notizie di reato non infondate, non deriva da considerazioni di opportunità relative alla singola notizia di reato, ma trova una ragione nel limite oggettivo alla capacità di smaltimento del lavoro dell'organismo giudiziario nel suo complesso e di questo ufficio in particolare».

La "circolare Zagrebelsky" generò accese discussioni, anche nell'opinione pubblica, perché svelava in tutta la sua evidenza, da un lato, l'incapacità del sistema di assicurare effettivamente l'obbligatorietà dell'azione penale ${ }^{6} \mathrm{e}$, dall'altro, il potere attribuito ai dirigenti degli uffici di definire le notizie di reato da perseguire in via prioritaria (attraverso specifiche "corsie preferenziali") ${ }^{7}$.

Fonte: CSM, dati 29 agosto 2014

${ }^{3}$ A livello nazionale, la scopertura del personale amministrativo si attesta al 18,64\%. I distretti con maggiori vacanze sono: Trento (23,3\% e 41,9\% nella sezione distaccata di Bolzano), Milano (25,1\%), Trieste $(19,4 \%)$ e Torino (19,2\%). Fonte: Ministero della giustizia, dati al 20.08.2014. Sul tema, si veda anche: Servizio Studi del Senato, Dati statistici relativi all'amministrazione della giustizia in Italia, n. 11, maggio 2013, 24 e ss.

"Si veda Zagrebelsky, Una "filosofia" dell'organizzazione del lavoro per la trattazione degli affari penali, in Cass. pen, 1989, 1615 ss.

${ }^{5} \mathrm{~V}$. ZaGrebelsky, Direttiva concernente criteri di priorità nella conduzione delle indagini preliminari, pubblicata, in Cass. pen., 1991, 362 e ss. Sul tema, si veda: ZaGrebelsky, Flusso delle notizie di reato, organizzazione delle risorse, obbligatorietà dell'azione penale, in Procure circondariali. Organizzazione del lavoro dei magistrati e rapporto con la polizia giudiziaria, in Quad. CSM, 1991, 163 e ss., e ID, Stabilire le priorità nell'esercizio obbligatorio dell'azione penale, in Il pubblico ministero oggi, $1994,105 \mathrm{e}$ Ss.

${ }^{6}$ La bibliografia su questi temi è molto vasta. Si veda, in particolare: FALCONE, Evoluzione del principio di obbligatorietà dell'azione penale, in Gius. proc., 1990, VI, 164 e ss., ICHINo, Obbligatorietà e discrezionalità dell'azione penale, in Quest. gius., 1997, 287 e ss., e Di Federico Obbligatorietà dell'azione penale e indipendenza del pubblico ministero, in Osserv. proc. pen., 2008, 1 e ss.

${ }^{7}$ Citando GaITO: «Va detto senza mezzi termini che al pubblico ministero è stata attribuita la titolarità di un potere discrezionale prognostico su una materia alquanto fluida, se non altro perché è lui stesso a determinare la maggiore o minore credibilità del materiale di indagine in diretta correlazione al maggio- 
Archivio Penale 2014, n. 3

Anche in assenza di una precisa prescrizione legislativa, sulla scia della circolare torinese, diversi dirigenti elaborarono documenti analoghi, sia nella forma che nella sostanza. Tali provvedimenti furono, di fatto, avvallati dal Consiglio Superiore della Magistratura. In particolare, il momento di svolta fu il procedimento disciplinare nei confronti di un magistrato della Procura circondariale di Roma che, in occasione del trasferimento ad altro ufficio, fu accusato di aver lasciato un notevole arretrato ${ }^{8}$. La sezione disciplinare del CSM formulò una decisione di ampia portata interpretativa, poi confermata in successive pronunce sul tema': «'impossibilità di tempestivamente esaudire la trattazione di tutte le notizie di reato implica che non ci si può sottrarre al compito di elaborare criteri di priorità: criteri che, una volta scontato come irragionevole il criterio che facesse mero riferimento al caso e alla successione cronologica della sopravvenienza delle notizie, non potrebbero non derivarsi, in ossequio alla soggezione anche dei pubblici ministeri solo alla legge, dalla gravità e/o offensività sociale delle singole specie di reati» (20 giugno 1997) ${ }^{10}$. Nello stesso anno, il Consiglio emanò anche una risoluzione, in risposta ad un ordine di servizio di un Procuratore, nella quale si stabiliva che: «la legge impone la registrazione di tutte le notizie di reato anche al fine della decorrenza dei termini e che eventuali disposizioni circa l'organizzazione del lavoro e le priorità di trattazione delle notizie di reato non possono essere generiche e discrezionali» (02 luglio 1997) ${ }^{11}$.

Su questa linea si muoveva anche l'art. 227 d.lgs. 19 febbraio 1998, n. $51^{12}$ che, allo scopo di aumentare la trasparenza dei criteri adottati negli uffici di procura e superare il rischio di scelte arbitrarie ${ }^{13}$, stabiliva espressamente che: «al fine di assicurare la rapida definizione dei processi pendenti [...] nella trattazione dei procedimenti e nella formazione dei ruoli di udienza, anche indipendentemente dalla data del commesso reato o da quella d'iscrizione del procedimento, si tiene conto della gravità e della concreta offensività del rea-

re o minore impegno profuso nell'espletamento dell'indagine stessa», in GAITO Accusa penale e ruolo del pubblico ministero, 1991, 22.

${ }^{8}$ Sul punto, si veda PaCileo, Pubblico ministero. Ruolo e funzioni nel processo penale e civile, 2011, 212 e ss.

${ }^{9}$ Si veda: CSM ord. nn. 50 del 2012 e 126 del 2012.

${ }^{10}$ Si veda: Cass., Sez. disc., 20 giugno, 1997, Note per l'autore: indicare imputato, in Cass. pen. 1998, 1489-1490.

${ }^{1}$ Sul tema della discrezionalità, si veda: FABRI, Discrezionalità e modalità di azione del pubblico ministero nel procedimento penale, in Polis, XI, 1997, 171 e ss. L'articolo è frutto di una ricerca empirica pluriennale condotta nelle Procure presso le Preture circondariali.

${ }^{12}$ C.d. "Riforma del giudice unico", pubblicata nella Gazzetta Ufficiale n. 66 del 20 marzo 1998.

${ }^{13}$ Si veda: VALENTINI, L'obbligatorietà dell'azione penale tra criteri di priorità e garanzia di uguaglianza, in Processo penale e costituzione, a cura di Dinacci, 2010, 127 e ss. 
Archivio Penale 2014, n. 3

to, del pregiudizio che può derivare dal ritardo per la formazione della prova e per l'accertamento dei fatti, nonché dell'interesse della parte offesa. Gli uffici comunicano tempestivamente al CSM i criteri di priorità ai quali si atterranno per la trattazione dei procedimenti e per la fissazione delle udienze». In questa fase storica, il dibattito si allargò ulteriormente, coinvolgendo non solo, come in passato, gli uffici requirent $i^{14}$, ma pure quelli giudicanti ${ }^{15}$. Anche sulla spinta di questa evoluzione, la materia dei criteri di priorità fu oggetto di svariati interventi del Legislatore, nel quadro della disciplina dettata dall'art. 132-bis disp. att. c.p.p. ${ }^{16}$.

\section{Il consolidarsi dell'indirizzo del CSM.}

A seguito della "Riforma del giudice unico" si consolidò l'indirizzo del CSM secondo cui, nella situazione di carenza di risorse, i criteri di priorità fossero compatibili con il principio di obbligatorietà dell'azione penale e rappresentassero una "leva" per assicurare eguaglianza di trattamento e buon andamento degli uffici requirenti e giudicant ${ }^{17}$.

La circolare sui criteri generali per le variazioni tabellari, che seguì la "Riforma del giudice unico" (12 aprile1999), definì in modo chiaro la posizione assunta dal CSM: «le indicazioni dell'art. 227 sono il segno di un orientamento che evidenzia la discutibilità di criteri meramente cronologici di trattazione dei processi, che possono avere come effetto quello di far si che la prescrizione si verifichi eventualmente in altri uffici. In tale prospettiva, appare pertanto necessario che in ogni distretto venga organizzata una "conferenza degli uffici" cui dovranno partecipare i dirigenti di tutti gli uffici giudicanti e requirenti [...]. Alla conferenza degli uffici competerà di elaborare soluzioni organizzative ed operative dirette ad assicurare la più sollecita definizione dei processi pendenti. La conferenza dovrà essere convocata per ciascun distretto [...] e dovrà costituire la sede naturale per la necessaria armonizzazione - pur nella considerazione delle diverse situazioni concrete - dei moduli organizzativi più idonei alla corretta applicazione dei criteri di cui all'art. 227. I verbali relativi

\footnotetext{
${ }^{14}$ Oltre alla "circolare Zagrebelsky", occorre ricordare, fra le altre, anche la direttiva del Procuratore della Repubblica presso la Pretura di Padova (04 ottobre 1993).

${ }^{15}$ In questo caso, si deve richiamare, fra le altre: la circolare del Presidente del Tribunale di Roma (07 maggio 1998) e quella del Presidente della Corte d'appello di Milano (12 dicembre 2008).

${ }^{16}$ Articolo introdotto dal d.l. n. 341 del 2000, convertito con modificazioni dalla l. n. 4 del 2001, modificato dal d.l. 92 del 2008, a sua volta convertito con modificazioni dalla l. n. 125 del 2008, modificato ancora dal d.l. n. 93 del 2013, a sua volta convertito con modificazioni dalla 1. n. 229 del 2013. Si veda anche la delibera del CSM del 13 novembre 2008.

${ }^{17}$ Sul punto, si veda: NiCOLÌ, Gli organi requirenti, in Ordinamento giudiziario. Uffici giudiziari, CSM e governo della magistratura, a cura di Di Federico, 2012, 137 e ss.
} 
Archivio Penale 2014, n. 3

ai lavori della conferenza dovranno essere allegati alla proposta di variazione tabellare che i dirigenti degli uffici sono tenuti a formulare in base alla presente circolare» ${ }^{18}$.

In particolare, la risoluzione del CSM in risposta alla nota del Ministero della giustizia «riguardante la possibilità di differenziare, rispetto agli altri, la tempistica dei processi penali destinati ad esaudirsi senza la concreta inflizione di una pena ricorrendo il beneficio dell'indulto» (09 novembre 2006) confermò questa impostazione, già richiamata in tutte le circolari sulle tabelle ${ }^{19}$ : «i dirigenti degli uffici (inquirenti e giudicanti) possono e devono, nell'ambito delle loro competenze in tema di amministrazione della giurisdizione, adottare iniziative e provvedimenti idonei a razionalizzare la trattazione degli affari e l'impiego, a tal fine, delle (scarse) risorse disponibili. [...] Tali scelte sono correttamente collocabili nell'ambito del sistema tabellare, assicurando in tal modo predeterminazione, uniformità e trasparenza e dimostrando la capacità e volontà dei dirigenti degli uffici di non rassegnarsi a una giurisdizione che produce disservizio, assumendosi la responsabilità di formulare progetti di organizzazione che [...] esplicitino le scelte di intervento adottate per pervenire a risultati possibili e apprezzabili».

L'indirizzo del CSM fu ribadito in occasione della famosa pronuncia sulla c.d. "circolare Maddalena" (10 gennaio 2007) - incentrata sull'idea di «accantonamento in attesa di tempi migliori», ai fini della non-trattazione di alcuni procedimenti $\mathrm{o}$, in subordine, della loro "archiviazione generosa" ${ }^{20}$. Al di là del merito di questa controversa decisione ${ }^{21}$ - che generò accese polemiche sia all'interno ${ }^{22}$ che all'esterno ${ }^{23}$ della magistratura - il CSM $^{24}$ confermò nuo-

\footnotetext{
${ }^{18}$ Circolare n. P-99-06928, Criteri generali per le variazioni tabellari.

${ }^{19} \mathrm{Si}$ veda, in particolare, la Circolare sulla formazione delle tabelle di organizzazione degli uffici giudiziari per il biennio 2002-2003, P-24710/2001 del 21.12.2001.

${ }^{20}$ Circolare n. 50/2007, in Quest. gius., 2007, 619 e ss. Sul tema, si veda anche: Valentini, L'obbligatorietà dell'azione penale tra criteri di priorità e garanzia di uguaglianza, in Processo penale e costituzione, a cura di Dinacci, cit., e Peri, Obbligatorietà dell'azione penale e criteri di priorità, in Forum di quaderni costituzionali.

${ }^{21}$ Inizialmente, la VII Commissione, nel valutare le «possibili conseguenze sul piano ordinamentale della circolare [...] e delle altre direttive emanate dai dirigenti degli uffici di Procura [Palermo e Busto Arsizio] in tema di trattazione dei procedimenti in conseguenza dell'applicazione dell'indulto» invitò il Procuratore di Torino a riconsiderare la circolare (07 maggio 2007).

${ }_{22} \mathrm{Si}$ veda: Magistratura Democratica, Cronache del Consiglio, Notiziario, in www.oldmagistraturademocratica.it.

${ }^{23}$ In particolare, la Camera Penale di Torino presentò numerose segnalazioni.

${ }^{24}$ Nella risposta a quesito del 10 ottobre 2007, il CSM volle chiarire che: «l'ambito di intervento del Consiglio nel settore della gestione degli affari giudiziari è sempre stato circoscritto alla sfera dell'organizzazione dell'attività giudiziaria, con esclusione di iniziative tese ad autorizzare - di diritto o di fatto - la mancata trattazione di alcuni procedimenti».
} 
Archivio Penale 2014, n. 3

vamente la volontà di promuovere il criterio di "economicità giudiziaria", attraverso «'adozione di moduli organizzativi adeguati, al fine di evitare o la mera casualità nella trattazione degli affari (e quindi il rifiuto di ogni razionalizzazione del lavoro) oppure l'adozione di criteri di fatto disomogenei all'interno dello stesso ufficio, non verificabili e perciò più esposti ad abusi e strumentalizzazioni» (15 maggio 2007) ${ }^{25}$.

\section{La "riscoperta” del dibattito.}

Il progressivo depauperamento delle risorse umane e materiali degli uffici giudiziari ha generato in questi ultimi anni una sorta di "riscoperta" dell'antico dibattito sui criteri di priorità. La necessità di gestire in modo oculato e razionale le poche risorse disponibili, ha spinto la stragrande maggioranza dei dirigenti a elaborare circolari sul tema ${ }^{26}$, anche per fronteggiare eventuali criticità interne, cali di produttività, incrementi delle sopravvenienze, allungamenti dei tempi di definizione e aumenti dell'arretrato ${ }^{27}$.

Di recente, il dibattito si è rianimato a seguito del decreto del Presidente del Tribunale di Roma (08 ottobre 2013) - approvato, in prima battuta, dal CSM (19 luglio 2013) ${ }^{28}$ - e dalla successiva nota del Procuratore della Repubblica presso il Tribunale ordinario di Roma (05 marzo 2014) ${ }^{29}$. Il caso di Roma, al pari di quello di Brescia - che sarà oggetto del prossimo paragrafo - ha ottenuto grande visibilità sui media, anche a livello nazionale ${ }^{30}$.

Qualche mese fa, il CSM è intervenuto di nuovo sulla questione (10 luglio 2014) $)^{31}$. L'ultima delibera si è aperta con una constatazione: «la necessità di individuare criteri di priorità si pone nelle situazioni di fatto, sempre più diffuse, caratterizzate dall'estrema difficoltà di procedere, nello stesso modo e secondo gli stessi tempi, alla trattazione di tutti gli affari pendenti presso un ufficio giudiziario». Oltre a riaffermare l'indirizzo consolidato del Consiglio, il documento ha sottolineato l'esigenza di collocare le scelte sui criteri di priorità nel quadro generale della disciplina tabellare, ovvero nelle tabelle di orga-

\footnotetext{
${ }^{25}$ Delibera adottata nel corso di una seduta straordinaria del plenum del Consiglio.

${ }^{26}$ Come segnalato anche dal Procuratore generale presso la Corte Suprema di cassazione in occasione del suo intervento alla cerimonia d'apertura dell'anno giudiziario 2014. Si veda: Ciani, Intervento nell'assemblea generale della corte sull'amministrazione della giustizia nell'anno 2013, in www.cortedicassazione.it.

${ }^{27}$ Si veda, per esempio: BARbuto, Argomenti dell'intervento orale del Presidente della Corte d'appello, inaugurazione dell'anno giudiziario 2014, in www.distretto.torino.giustizia.it.

${ }^{28}$ Prot. P14531/2013.

${ }^{29}$ Circolare 4-bis del 05.03.2014, protocollo n. 550.

${ }^{30}$ Si veda: Corriere della Sera, Processi a numero chiuso a Roma, 07 marzo 2014.

${ }^{31}$ Prot. P12858/2014.
} 
Archivio Penale 2014, n. 3

nizzazione dell'ufficio e delle tabelle infradistrettuali, attraverso una «procedura partecipata, che è opportuno sia estesa oltre i confini del rapporto tra uffici inquirenti e giudicanti corrispondenti, per coinvolgere tutti gli uffici di ogni singolo distretto avvinti da rapporti di tipo funzionale». Il Consiglio ha incoraggiato altresì i capi ufficio a inserire le misure organizzative: «in una strategia complessiva di lungo periodo, sottratta alle mutevoli contingenze costituite da picchi di flusso, che possono essere, invece, gestiti in modo più efficace ove considerati in ambiti temporali più dilatati».

\section{Il decreto del Tribunale ordinario di Brescia.}

Il decreto del Presidente del Tribunale ordinario di Brescia n. 2491 del 19 maggio 2014 ha definito i criteri di priorità vigenti presso le Sezioni penali della corte. Il provvedimento si inserisce nella lunga tradizione di interventi sul tema, sia ad opera del CSM che di altri uffici giudicanti e requirenti. La nota segue temporalmente, pur senza citarla, nel segno di una netta discontinuità, la direttiva del precedente Presidente (22.10.2009) ${ }^{32}$.

La circolare 2491/2014 richiama la delibera del Consiglio Superiore, adottata a seguito dell'audizione dall'attuale Presidente del Tribunale, nella quale: «si da atto del sottodimensionamento della pianta organica [...] con "sollecitazione" al Ministro affinché valuti l'opportunità - che nella situazione descritta assume i contorni della vera e propria necessità - di procedere a un aumento significativo dell'organico» (13 marzo 2014).

Il provvedimento, richiesto dagli stessi magistrati dell'ufficio ${ }^{33}$, risulta collegato al programma di gestione 2014 e al documento organizzativo generale (DOG) relativo al progetto tabellare 2014/2016 e riporta allegata una scheda sui flussi processuali - in particolare, citazioni dirette $e x$ art. 550 c.p.c. e opposizioni a decreto penale - elaborata dalla cancelleria centrale delle sezioni penali.

Nel merito, vista l'urgenza di governare il progressivo aumento delle richieste di giudizio dibattimentale ${ }^{34}$, il decreto del Presidente del Tribunale di Brescia individua una serie di priorità nella gestione di udienze e sopravvenienze. Nello specifico, questo provvedimento stabilisce che: «impregiudicata la fissa-

\footnotetext{
${ }^{32}$ Direttiva in materia di applicazione degli artt. 2-bis e 2-ter d.l. n. 92 del 2008, convertito con modificazioni nella l. n. 125 del 2008, in cui si legge: «non è compito di questa Presidenza individuare quali processi e in quale ordine siano da considerarsi a trattazione prioritaria, poiché gli stessi sono stati con chiarezza indicati dal Legislatore nell'art. 2-bis, co. 1».

${ }^{33}$ Citando il testo del decreto n. 3573 del 2014: "i Presidenti delle sezioni penali da tempo hanno auspicato interventi nell'indicata direzione, più volte sollecitata dai giudici nel corso delle consuete riunioni sezionali".

${ }^{34}$ Dai dati della cancelleria, negli ultimi anni le richieste di giudizio dibattimentale sono aumentate sensibilmente: 1.972 (2009), 2.290 (2010), 3.330 (2011), 3.999 (2012), 6.001 (2013).
} 
Archivio Penale 2014, n. 3

zione prioritaria dei processi di cui all'art. 132-bis disp. att. c.p.p., nei dodici mesi successivi all'entrata in vigore del presente provvedimento ${ }^{35}$, tutte le udienze di prima comparizione dei processi a citazioni diretta e da opposizione a decreto penale di condanna siano destinate alla fissazione di quelli aventi ad oggetto...». Il decreto elenca le priorità cui dovranno attenersi i magistrati delle sezioni penali - fra cui, per esempio, «i fatti costituenti delitto, e tra essi, per primi, i reati di lesioni colpose da infortunio sul lavoro e maltrattamenti familiari» - e definisce altresì una serie di eccezioni generali ${ }^{36}$.

\section{Riflessioni conclusive.}

Questo articolo si è occupato di tratteggiare i confini del vasto dibattito sui criteri di priorità negli uffici requirenti e giudicanti italiani, approfondendo, in particolare, il caso del recente decreto del Presidente del Tribunale ordinario di Brescia.

In conclusione, è opinione ampiamente diffusa - condivisa da buona parte degli studiosi di ordinamento giudiziario ${ }^{37}$, così come da numerosi esponenti della magistratura $^{38}$ e dell'avvocatura ${ }^{39}$ - che il principio dell'obbligatorietà dell'azione penale - almeno come conosciuto fino a oggi - andrebbe ripensato. Pur nella consapevolezza che non è questa la sede per approfondire tali riflessioni, appare evidente, soprattutto nella situazione di difficoltà in cui versa il sistema italiano, che occorra una riforma di ampio respiro, capace di tenere conto delle esperienze degli altri Paesi di tradizione democratica ${ }^{40}$. Qualsiasi intervento in materia di criteri di priorità dovrebbe, infatti, coniugare l'esigenza di salvaguardare l'indipendenza della magistratura con la necessità di appianare possibili differenze territoriali nel modo di concepire ed esercitare l'azione penale, garantendo in tal modo, a tutti gli effetti, l'eguaglianza dei cittadini di fronte alla legge. Una riforma di questo tipo potrebbe generare delle economie di scala, evitando sprechi di risorse, in un'ottica di qualità,

\footnotetext{
${ }^{35}$ Come si legge nella parte conclusiva del medesimo documento: «il presente decreto è provvisoriamente esecutivo subordinatamente al parere favorevole unanime del Consiglio Giudiziario, salva la deliberazione del CSM».

${ }^{36}$ Si rimanda al testo integrale del decreto, in questa Rivista online.

${ }^{37} \mathrm{Si}$ vedano, in particolare, i contributi contenuti in GAITO, Accusa penale e ruolo del pubblico ministero, cit., e Kostoris, Per un'obbligatorietà temperata dell'azione penale, in Riv. dir. proc., 2007, 878 e ss.

${ }^{38} \mathrm{Si}$ ricorda, ad esempio, il c.d. "Manifesto dei 4 procurator1". Fonte: Corriere del mezzogiorno, Bisogna scegliere i reati su cui indagare, 02 luglio 2010.

${ }^{39} \mathrm{Di}$ recente, anche il Consiglio Nazionale Forense si è espresso sul tema. Si veda: Il Sole 24 Ore, $S_{U-}$ perare il dogma dell'azione penale obbligatoria, 23 agosto 2014.

${ }^{40}$ Si veda: Di Federico, L'indipendenza e responsabilità del pubblico ministero italiano in prospettiva comparata, in Giurisprudenza Italiana, 2009.
} 
Archivio Penale 2014, n. 3

efficienza, trasparenza ${ }^{41}$ e accountability degli uffici giudiziari ${ }^{42}$.

"In particolare, pensando a modalità di coinvolgimento delle istituzioni del territorio, come avviene in altri sistemi, fra cui quello olandese.

${ }^{12}$ Anche attraverso l'introduzione di indicatori sulle condizioni socio-economiche del territorio in cui opera la corte (es. reddito della popolazione, denunce, reati, tasso di criminalità, ecc.). 